\title{
Abdominal Cocoon: An Unusual Cause of Intestinal Obstruction
}

\author{
Tapesh Kumar Paul ${ }^{1}$, Russel Ahmed Khan Lodi ${ }^{2}$, Shayda Ali ${ }^{3}$, \\ Mohammad Arman Zahed Basunia ${ }^{4}$, Hasan Md. Abdur Rouf 5
}

\begin{abstract}
Abdominal cocoon, the idiopathic form of sclerosing encapsulating peritonitis, is a rare condition of unknown etiology that results in an intestinal obstruction due to total or partial encapsulation of the small bowel by a fibrocollagenous membrane. The early clinical features are nonspecific, are often not recognized and it is difficult to make a definite pre-operative diagnosis. Clinical suspicion may be generated by the recurrent episodes of small intestinal obstruction combined with relevant imaging findings and lack of other plausible etiologies. Surgery is important in the management of this disease. Careful dissection and excision of the thick sac with the release of the small intestine leads to complete recovery in the vast majority of cases. Here a case of abdominal cocoon in a 45 years old male is presented due to its rarity and difficulty in preoperative diagnosis.
\end{abstract}

Keywords: Abdominal cocoon; intestinal obstruction.

Delta Med Col J. Jan 2017;5(1):49 - 52

\section{Introduction}

Abdominal cocoon is a rare condition that refers to total or partial encapsulation of the small bowel by a fibrocollagenous membrane causing clustering of the bowel which was first described by Owtschinnikow in 1907 as "peritonitis chronica fibrosa incapsulata"1 and subsequently termed "abdominal cocoon" by Foo ${ }^{2}$ in 1978. The exact cause and pathogenesis of the abdominal cocoon syndrome (or idiopathic encapsulating peritonitis) have not been elucidated though there are several theories. The majority of the cases are reported from tropical and subtropical climate belts of the world and usually occur in female. ${ }^{3}$ It commonly presents as intestinal obstruction, weight loss or abdominal mass and in some cases it may be asymptomatic.

\section{Case Report}

A 45 year old male presented in the emergency with complaint of severe colicky lower abdominal pain for 6 hours after taking mango. Patient gave past history of 6-7 similar type of attacks with low intensity within last 3-4 years which was managed

1. Associate Professor, Dept. of Surgery, Delta Medical College, Dhaka, Bangladesh.

2. Resident Surgeon of Surgery, Delta Medical College, Dhaka, Bangladesh.

3. Registrar of Surgery, Delta Medical College, Dhaka, Bangladesh.

4. Registrar of Surgery, Delta Medical College, Dhaka, Bangladesh

5. Professor of Surgery, Delta Medical College, Dhaka, Bangladesh.

Correspondence: Dr. Tapesh Kumar Paul, email: tapeshpaul@yahoo.com 
conservatively by injecting some drugs. Patient was afebrile and gave history of single episode of vomiting. He was a known case of pulmonary tuberculosis and completed treatment at the age of 18 years.

During admission patient was haemodynamically stable. Examination of the abdomen revealed an intra-abdominal mass occupying the upper part of right iliac fossa, right lumbar and umbilical region. Feeling beneath the mass in right iliac fossa was empty. Diffuse tenderness along with hyperactive bowel sounds was present. Digital rectal examination revealed no abnormality. Findings of other systemic examinations were normal.

Plain X-ray abdomen in the erect posture showed few gas fluid levels at right iliac fossa and umbilical region and no gas under the dome of diaphragm (Fig. 1). On abdominal ultrasonography bowel loops seemed to be dilated having no collection in right iliac fossa. Complete blood count revealed neutrophilic leucocytosis.

Our differential diagnoses were intestinal obstruction due to intussusception or ileocecal mass. But nothing was conclusive with these investigations and further radiological investigations were not suitable for the patient in this condition.

Exploratory laparotomy through a mid line incision was performed. At first site it seemed that it was a caecal dilatation. But careful evaluation found that there was no small intestine in infracolic region. Then duodeno-jejunal junction was explored and it was confirmed that the small intestine entered into a sac which contained almost whole of the small intestine along with the mesentery. After that the sac was opened and coils of intestines were released by adhesiolysis (Fig. 2). Complete excision of sac was not possible because stripping of it might make the intestinal wall raw which in future could lead to more adhesion and thereby cause intestinal obstruction.
The white fibrous membrane pieces were sent for histopathology examination which revealed fibrocollagenous tissue, with congested blood vessels and infiltration of inflammatory cells. Post operative course was uneventful and patient was discharged on 8th post operative day.

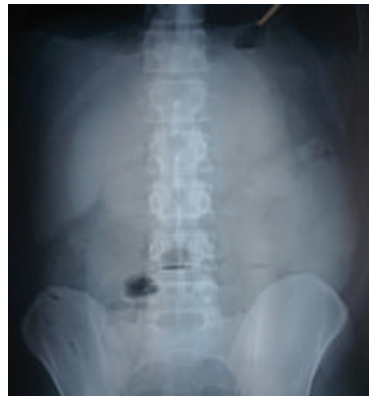

Fig. 1: Plain X-Ray abdomen of the patient

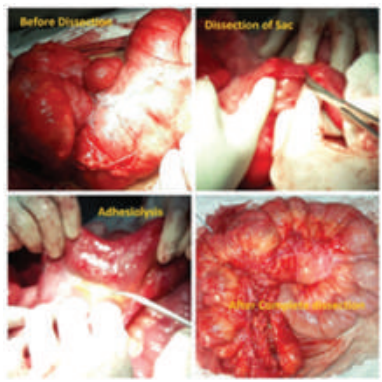

Fig. 2: Showing dissection of capsule of the patient

\section{Discussion}

Sclerosing encapsulating peritonitis is classified as primary or idiopathic and secondary when it has a definitive cause. Primary form has unknown cause, so many theories have been put forward like subclinical peritonitis leading to formation of cocoon. Idiopathic form is known as abdominal cocoon. Tu et al. reported a retrospective study of 203 cases of abdominal cocoons where mean age was 33 years, male to female ratio 1.2:1, features of bowel obstructionin $72.4 \%$, abdominal mass in $26.1 \%$ cases. Only $3 \%$ of cases were diagnosed preoperatively. Enterolysis was done in $84.7 \%$, bowel resection in $16.7 \%$, appendectomy in $25.1 \%$ of cases. Post operative recurrent obstruction was found in $11 \%$ and mortality was $5.4 \%{ }^{4}$ It is common in females which may be due to retrograde menstruation causing peritonitis, or cell mediated tissue damage caused by gynaecological infections. Brijender et.al reported 18 cases of abdominal cocoon where more than half of them had abdominal tuberculosis features per-operatively which were later confirmed by histopathological examination post operatively. ${ }^{5}$ Developmental conditions like greater omentum 
hypoplasia, mesenteric vessel malformation may also play role in its aetiology. Secondary causes are shunt placement for refractory astites ${ }^{1}$, systemic lupus erythematosus, continuous ambulatory peritoneal dialysis ${ }^{6}$, use of povidone iodine in abdominal irrigation ${ }^{7}$ and use of practolol $^{8}$. Other causes are abdominal tuberculosis, sarcoidosis, endometriosis, protein $\mathrm{S}$ deficiency, dermoid cyst rupture, cirrhosis, liver transplantation, gastrointestinal malignancy, Mediterranean fever, and intraperitoneal chemotherapy.

Clinically patient may present with recurrent abdominal pain, vomiting, anorexia, weight loss, and episodes of subacute bowel obstruction. But these features may be misleading to diagnosis. X-ray abdomen may show small bowel loops enclosed in cocoon of peritoneum causing proximal bowel dilatation or dilated bowel loop lying in a concertina fashion giving cauliflower sign. ${ }^{9}$ CT scan remains the gold standard in diagnosis of abdominal cocoon. It demonstrates thickened membrane encasing small bowel loops, thickened bowel loops, tethering or matting of bowel or peritoneal calcification. ${ }^{10}$ Fibrosis of mesentery causes clumping and tethering of bowel loops resulting in gingerbread man sign.

Despite the advancement of radiological investigations most of the cases are diagnosed after laparotomy. Bo et al. reported that out of 24 cases of abdominal cocoon only four cases were diagnosed by radiological imaging while 20 cases were diagnosed at laparotomy. ${ }^{11}$ Differential diagnoses are internal hernias, intussusception, and localized peritoneal adhesions. Based upon the surgical findings abdominal cocoon can be classified into three types, Type- 1 partial bowel is encased in membrane, Type- 2 entire bowel is encapsulated by membrane, Type- 3 entire intestine and other organs like ovary, appendix, caecum, colon are encased by membrane. ${ }^{11}$

Surgery includes stripping of membrane, release of small intestine and in some cases enterolysis, and bowel resection plays main stay of treatment We did surgical stripping and enterolysis. Treatment of secondary disease is directed to controlling the primary disease. Laparoscopic excision is emerging as treatment of abdominal cocoon. ${ }^{12}$ Common complications after surgery are intra-abdominal infections, perforation of bowel and in some cases entero-cutaneous fistula.

\section{Conclusion}

Abdominal cocoon is a rare disease with unknown etiology. A high index of suspicion is required in diagnosing and surgery plays the definitive procedure of treatment.

\section{References}

1. Owtschinnikow PJ. Peritonitis Chronica Fibrosa Incapsulata. Archiv für Klinische Chirurgie. 1907;83:623-34.

2. Foo KT, Ng KC, Rauff A, Foong WC, Sinniah R. Unusual Small Intestinal Obstruction in Adolescent Girls: The Abdominal Cocoon. Br J Surg. 1978;65(6):427-30.

3. Kumar M, Deb M, Parshad R. Abdominal Cocoon: Report of a Case. Surg Today. 2000;30(10):950-53.

4. Tu JF, Huang XF, Zhu GB, Liao Y, Jiang FZ. Comprehensive Analysis of 203 Cases with Abdominal Cocoon. Chinese Journal of Gastrointestinal Surgery. 2006;9(2):133-35.

5. Singh B, Gupta S. Abdominal Cocoon: A Case Series. International Journal of Surgery. 2013;11(4):325-28.

6. Okada K, Onishi Y, Oinuma T, Nagura Y, Soma M, Saito S, et al. Sclerosing Encapsulating Peritonitis: Regional Changes of Peritoneum. Nephron. 2002;92:481-83.

7. Keating JP, Neill M, Hill GL. Sclerosing Encapsulating Peritonitis after Intraperitoneal Use of Povidone Iodine. ANZ Journal of Surgery. 1997;67(10):742-44. 
8. Thompson RPH, Jackson BT. Sclerosing Peritonitis due to Practolol. British Medical Journal. 1977;1:1393-94.

9. Tombak MC, Apaydin FD, Colak T, Duce MN, Balci Y, Yazici M, et al. An Unusual Cause of Intestinal Obstruction: Abdominal Cocoon. American Journal of Roentgenology. 2010;194(2):176-78.

10. Loughrey GJ, Hawnaur JM, Sambrook P. Case Report: Computed Tomographic Appearance of Sclerosing Peritonitis with Gross Peritoneal
Calcification. Clinical Radiology. 1997;52(7): 557-58.

11. Wei B, Wei HB, Guo WP, Zheng ZH, Huang Y, Hu BG, et al. Diagnosis and Treatment of Abdominal Cocoon: A Report of 24 Cases. The American Journal of Surgery. 2009;198(3):348-53.

12. Ertem M, Ozben V, Gok H, Aksu E. An Unusual Case in Surgical Emergency: Abdominal Cocoon and Its Laparoscopic Management. Journal of Minimal Access Surgery. 2011;7(3):184-86. 